\title{
A importância da redução de custos e a utilização da metodologia Kaizen como ferramenta auxiliar nesse processo
}

C aroline R odrigues B atista

E llen C ristina B raga M odesto

$\mathrm{M}$ ariane do $\mathrm{N}$ ascimento Silva

M ônica Pereira da Silva

D iscentes do C urso de Ciências C ontábeis - UB M C entro Universitário de B arra M ansa

W anderli A ntônio de 0 liveira M BA em C ontroladoria e F inanças, UN IF O A D ocente $C$ urso de $C$ iências C ontábeis - UBM C entro Universitário de Barra Mansa 


\section{Resumo}

A globalização e a acessibilidade de informações e recursos vêm pressionando e impulsionando as organizações na busca de ferramentas que possibilitem maior competitividade e, consequentemente, menor custo. Os avanços tecnológicos, cada vez mais, exigem das empresas um monitoramento minucioso dos mercados onde as mesmas encontram-se inseridas, atualizando a metodologia de condução de seus processos e evitando a obsolescência de seus produtos e serviços. A inda nesse contexto, muitas empresas desconhecem os custos totais inerentes à produção e prestação de serviços, tornando seu negócio economicamente inviável para um mercado em constante evolução e exigente em termos de qualidade e procedência de seus produtos. Portanto, este artigo busca apresentar a importância da contabilidade de custos dentro de uma empresa, a fim de obter controle dos mesmos, auxiliando a gestão financeira para tomada de decisões, adotando a metodologia K aizen como uma ferramenta auxiliar para a redução dos custos de produção. 0 desenvolvimento desse sistema tem por base a busca contínua da minimização dos custos, associados aos processos produtivos da empresa, mantendo qualidade de produtos e serviço final. A implementação do sistema em questão envolve algumas etapas que formam a base para as premissas de custeio da organização, bem como a necessidade do envolvimento desde a alta gerência até a mão de obra direta. A metodologia Kaizen possui diversas aplicabilidades no ambiente organizacional e, neste artigo, poderá ser aplicada de várias formas, como na melhoria na manufatura de produtos e prestação de serviços.

Palavras-chave: M etodologia Kaizen. C ontrole de custos. D esperdícios. 


\section{Abstract}

The globalization and the accessibility of information and resources have been pushing and impelling organizations in the search for tools that allow greater competitiveness and, consequently, lower costs. The technological advances, increasingly, require companies to carefully monitor their markets, updating the methodology of conducting their processes and avoiding the obsolescence of their products and services. Still in this context, many companies are unaware of the total costs inherent in the production and provision of services, making their business economically unviable for a market that is constantly evolving and demanding in terms of the quality and products' origin. Therefore, this article seeks to present the importance of cost accounting within a company, in order to obtain control of them, helping the financial management for decision making, adopting the Kaizen methodology as an auxiliary tool to reduce production costs. The development of this system is based on the continuous search for cost reduction, associated to the productive processes of the company, maintaining quality of its products and final service. The implementation of this system involves a number of steps that form the basis for the organization's costing assumptions, as well as the need for involvement from top management to direct labor. The Kaizen methodology has several applicabilities in the organizational environment and, in this article, can be applied in several ways, as in the improvement in the manufacture of products and services.

Keywords: K aizen methodology. Cost control. W astage. 


\section{Introdução}

No cenário mundial, as relações de dependência da economia e o ambiente competitivo em que as empresas estão inseridas passam por uma grande pressão, o que torna necessária a redução de preços e margem de lucro. Para que isso ocorra, é vital a minimização de custos, permitindo que a empresa alcance sucesso e continuidade, e dessa forma mantenha-se no mercado.

A ssim, a contabilidade de custos torna-se essencial para a organização, sendo a mesma responsável por entender gastos relacionados à produção de bens e serviços, fornecendo informações fundamentais às atividades pertencentes ao planejamento, controle e à tomada de decisões.

D e acordo com M egliorini (2001), quando uma empresa apura seus custos, atende as exigências legais em relação à apuração de resultados de suas práticas, além de obter 0 entendimento dos seus custos para o auxílio adequado no momento da tomada de decisões, exercendo seus controles.

Um monitoramento de custos eficiente e que acompanhe continuamente as alterações oriundas do cenário vivido pela empresa é necessário, não só para conhecer custos, mas para buscar a sua diminuição. Para se alcançar um acompanhamento de custos eficiente, é necessário que seja feita uma reavaliação dos objetivos da empresa frente ao mercado, ou seja, fazer uma análise de até quanto o mercado pagaria por esse produto ou serviço, e com base nessa informação, estabelecer o padrão para o alcance de suas metas.

Existem diversos métodos de apurações de custos com aplicações e critérios diferentes dos quais este artigo irá tratar, pois a implementação de sistemas e estratégias que possam contribuir para o controle de custos e redução dos mesmos faz-se necessária para preparar a empresa e incorporá-la para o futuro com a intenção de obter orçamentos extremamente saudáveis.

$\mathrm{N}$ esse contexto, a metodologia ou filosofia Kaizen é uma ferramenta auxiliar na diminuição e monitoramento dos custos que estão presentes nas etapas do processo, permitindo que se aproxime ao máximo do lucro que se deseja obter (MONDEN, 1999).

E ste artigo tem como objetivo apontar a importância da filosofia Kaisen como ferramenta para diminuição e acompanhamento das práticas produtivas da empresa. 
As empresas precisam priorizar e se empenhar em melhorar constantemente seus processos, o que significa estar diariamente atribuindo alternativas e mudanças necessárias para a execução dos mesmos processos a custo menor, porém com o mesmo padrão de qualidade.

É fundamental que a empresa se adeque à realidade e busque continuamente reduzir custos, adotando práticas de monitoramento de custos até o limite de alcance do retorno esperado, ou que, no mínimo, satisfaça a necessidade da organização.

Com 0 aumento da população mundial, os fornecedores de bens e serviços se viram na condição de produzirem mais e venderem a preços mais baratos para conseguirem atingir a todas as classes, denominadas como A, B, C, D e assim por diante. $\mathrm{E} \mathrm{m}$ tese, quanto mais alta a camada social da população, menores são os volumes e mais elevados são os preços.

Diante desse crescimento, os fabricantes, comerciantes e seus adversários concorrentes começaram a buscar formas mais inteligentes de reduzir custos de processos para ofertar produtos e serviços a preços mais competitivos, os chamados preços mais baixos.

Reduzir custos excessivos é essencial para as empresas sem, contudo, eliminar o potencial de crescimento futuro e sem, tampouco, reduzir sua estrutura operacional existente. O s custos que não agregam valor ao cliente, de forma direta ou indireta, são dignos de serem eliminados.

Para isso é necessário que o corpo gerencial da empresa tenha habilidade suficiente para identificar quais são esses custos passíveis de serem cortados, sem prejudicar o processo e a estrutura operacional existentes, necessários para dar continuidade ao negócio e preservar o atendimento de clientes.

O s custos precisam ser gerenciados permanentemente e com visão de longo prazo. N ão se pode deixar de lado o planejamento dos custos fixos e variáveis, bem como os relatórios comparativos entre o orçado e o real, pois o preço sempre é chamado de pré-cálculo por causa do natural planejamento, feito antes da liberação da chamada tabela de preços.

Para ingressar no mundo dos negócios, em um ambiente altamente competitivo, em que o cenário se altera constantemente, obter o conhecimento dos custos de suas atividades e dominar a aplicabilidade dos mesmos se tornaram condições básicas. 
Por meio da filosofia kaizen, as empresas conseguem reduzir seus custos e aumentar sua produtividade. Esse resultado ocorre, porque essa metodologia permite uma melhoria contínua e diversas mudanças em todos os processos da empresa. Para que a competitividade desejada fosse al cançada, seria necessário buscar, identificar e eliminar os desperdícios de um determinado processo.

\section{Método Kaizen}

O Kaizen foi a ferramenta utilizada para alcançar competitividade. A palavra Kaizen é originária do Japão e provém da união de kai (mudar) e zen (melhor), ou seja, melhoria contínua que incide nos processos de manufatura, engenharia, apoio a processos de negócios e gestão.

T anto nas empresas como na vida pessoal é necessário sempre reduzir custos ou eliminar desperdícios, pois em se tratando de redução de custos e aumento de produtividade, as pessoas optam por pagar menos pelos bens e serviços adquiridos (FERREIRA, 2007; SANT OS, 2017).

U m ponto importante sobre o Kaizen é que o mesmo não foi criado com 0 objeto de trabalhar com custo de forma específica, e, é comum associar o Kaizen somente a custos, porém, é importante assimilar que esse conceito pode ser utilizado em diversas situações como uma ferramenta que permite melhorar desempenhos, por isso é útil em diversos setores, seja vida pessoal, comercial ou industrial.

Para Imai (1994):

Kaizen significa melhoramento. $M$ ais que isso, significa contínuo melhoramento na vida pessoal, na vida domiciliar, na vida social e na vida no trabalho. Q uando aplicado no local de trabalho, KAIZEN significa contínuo melhoramento envolvendo todos - tanto os gerentes quanto os operários (I M A I, 1994, p.4).

Existem várias alternativas dentro do Kaizen que podem auxiliar 0 setor responsável pelos custos. É possível visualizá-las por meio do guarda-chuva de Kaizen, apresentado abaixo: 


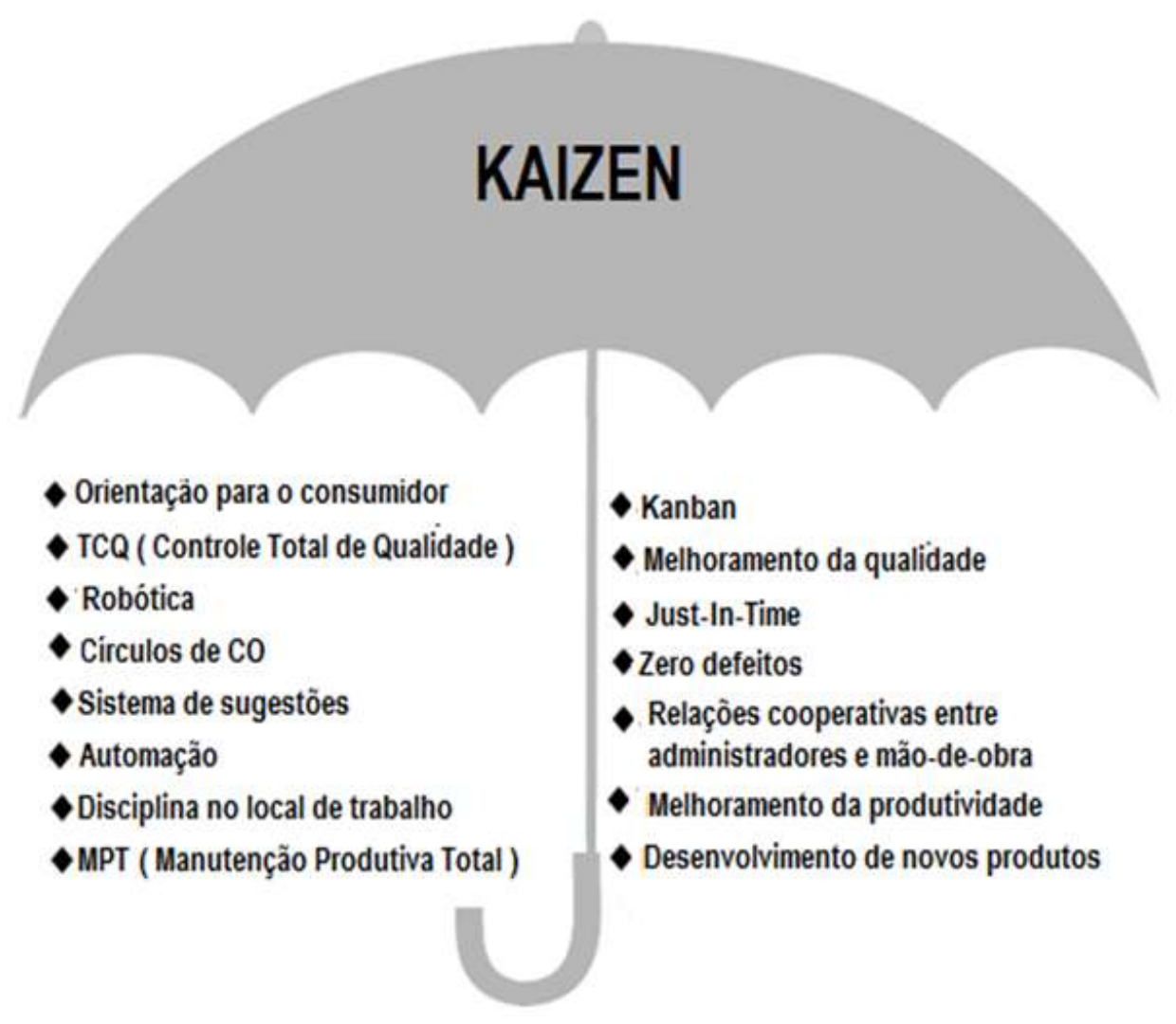

Figura 1. G uarda-chuva do K aizen

FONTE : Imai (1994, p.4)

Segundo Monden (1999, p. 221), Kaizen "significa manter os níveis correntes de custo e trabalhar sistematicamente para reduzir os custos aos valores desejados".

Q uando se aplica o monitoramento constante de processos paralelamente às abordagens contantes do guarda-chuva do Kaizen, é possível alcançar bons resultados em relação à diminuição e eliminação dos custos que variam com a produção, possibilitando também adoção de práticas para diminuir as perdas.

$N$ ormalmente são estabelecidas metas para 0 custo ideal de um processo e a metodologia Kaizen pode ser utilizada como uma ferramenta de apoio para alcançar tais objetivos em relação a esse custo ideal.

A gndal (2009) aponta que:

Engenharia de valor (EV) e análise de Valor (AV, também "kaizen" ou "melhoramento contínuo") são duas importantes técnicas mencionadas como importantes ferramentas para e realização do custo-alvo (AGN D AL , 2009, p. 29). 
Para M esquita e Allipradini (2003), como as mudanças atual mente ocorrem muito rapidamente, é de grande importância a adoção de um monitoramento constante e intenso dos processos, devendo os pensamentos, todos os esforços e ações se concentrarem nesse monitoramento, de forma que se possa acompanhar as constantes mudanças.

Segundo pesquisas e estudos de F arris et al. (2009), essa metodologia se traduz num mecanismo capaz de auxiliar no alcance de melhores resultados organizacionais, relativos a mudanças na execução do trabalho e no desenvolvimento e capacitação do corpo de funcionários.

U ma implantação de sucesso pode resultar no alcance de grandes benefícios, 0 que se comprova segundo L eite (2007), ao citar que empresas como a Toyota M otor, TokaiRika, A isin, A ichi, D ai N ipponT oryo, JVC, T etra-Pak adotaram a metodologia e foram bem-sucedidas.

Como mencionado, a T oyota foi a primeira a implementar o sistema Kaizen no seu processo de produção e sua finalidade segundo I mai (1994) é reduzir continuamente os custos gerados no decorrer da produção diminuindo-os e se aproximando ao máximo do custo determinado como meta para o alcance dos objetivos da organização.

De acordo com Cooper e Slagmulder (2003), deve-se trabalhar com a metodologia Kaizen e o custo que se espera do processo juntos, porém, em momentos diferentes, ou seja, no momento do processo, aplicar o Kaizen, e no momento de estudo e projeção do produto, o custo que seria a meta ou o ideal para o processo, e assim se obter custos menores no decorrer dos processos.

É preciso ainda uma análise em relação a mudanças tecnológicas que ocorrem atualmente, pois diversas ou mesmos todas as áreas da empresa estão sofrendo os impactos e passando por mudanças devido à tecnologia, e o setor que monitora os custos também está dentro desse contexto, uma vez que a tecnologia tem contribuído com soluções inteligentes, servindo como ferramenta útil à tomada de decisões e como um recurso para que informações possam ser armazenadas e acessadas com rapidez e eficiência (C O O PER; SLA G M ULDER, 2003). 


\section{Visão do Sistema ou Metodologia Kaizen}

O custo ou metodologia kaizen se distingue dos demais sistemas de custeio, nas suas caracterizações e nos seus métodos, referente ao monitoramento dos custos, porque segundo M onden (1999) trata-se de um sistema que busca:

$\checkmark$ Reduzir, controlar ou mesmo eliminar custos que se objetiva em impulsionar a diminuição de custos reais de um determinado produto para um estágio menos elevado em relação aos custos-padrão;

$\checkmark$ Realiza controle buscando impactar reduções ou eliminações de custoalvo;

$\checkmark$ As manufaturas sofrem constantes transformações com a intenção de reduzir custos; metas de controle, redução ou eliminação (quando possível) são determinadas todo mês, ideia esta que busca acabar com as distorções entre os lucros estabelecidos como meta e lucros que foram estimados no projeto, enquanto um sistema baseado no custeio-padrão faz só uma, ou duas vezes;

$\checkmark \quad$ No decorrer do ano comercial, são acompanhadas atividades kaizen, ou seja, a busca em melhorar constantemente o processo e assim controlar e minimizar os custos-alvo ou a meta já determinada;

$\checkmark$ A companhamento e análise referente a diferenças entre custos-alvo já determinados como meta para o processo e os custos realmente gerados no mesmo; medidas corretivas são tomadas, quando as metas estabelecidas para diminuição dos custos não são alcançadas, realizando então investigações.

M onden (1999) afirma ainda que, um sistema de custos baseado na metodologia kaizen envolve não só o sistema contábil e administrativo da empresa, mas também as atividades kaizen aplicadas ao nível do chão de fábrica (JIT, TQ M, entre outros).

D esse modo, Sakurai (1997) assinala que o custo kaizen engloba atividades de redução, diminuição ou mesmo eliminação de custos que estão divididas em dois tipos: entende-se pelo primeiro as atividades concentradas à diminuição de custos presentes em cada produto e pelo segundo tipo, as atividades relacionadas à diminuição de custos 
em relação aos departamentos, por período. 0 quadro abaixo apresenta as características relativas aos sistemas baseados no custeio tradicional:

Q uadro 1. Características relativas aos sistemas baseados no custeio tradicional

\begin{tabular}{|c|c|}
\hline SISTEMAS & CARACTERÍSTICAS \\
\hline A bsorção & $\begin{array}{l}\text { O rigem no chão de fábrica } \\
\text { O rientado para o controle da produção } \\
\text { Produção como elemento gerador de riqueza } \\
\text { L ucro unitário como parâmetro de análise } \\
\text { Projetado para monitorar a produção em termos de volume, tempo e custo. }\end{array}$ \\
\hline Direto & $\begin{array}{l}\text { O rigem gerencial } \\
\text { O rientado para as funções financeiras e de marketing } \\
\text { V endas como elemento gerador de riqueza } \\
\text { Só os custos variáveis são imputados no produto } \\
\text { O s custos fixos pertencem à estrutura } \\
\text { M argem de contribuição unitária como parâmetro de análise } \\
\text { Ê nfase na análise da relação custo-volume lucro }\end{array}$ \\
\hline UP & $\begin{array}{l}\text { O rientado para a produção } \\
\text { M ensuração da produtividade para empresas multiprodutoras } \\
\text { R elação constante entre os potenciais produtivos das seções homogêneas } \\
\text { Padrão potencial para a mensuração de desempenho } \\
\text { U nidade abstrata (UP) para estabelecer equival ência entre custos de produtos } \\
\text { L ucro unitário como medida de desempenho }\end{array}$ \\
\hline$A B C$ & $\begin{array}{l}\text { O rientado para o processo } \\
\text { A plicável a toda a cadeia de valor } \\
\text { A s atividades consomem recursos e os produtos consomem atividades } \\
\text { I dentifica as atividades que agregam valor aos produtos } \\
\text { R ateia os custos indiretos com critérios multidimensionais } \\
\text { I dentifica responsabilidade pelas atividades que mais consomem recursos }\end{array}$ \\
\hline M eta/A Ivo & $\begin{array}{l}\text { O rientado para o processo } \\
\text { A plicável a toda a cadeia de valor } \\
\text { Instrumento gerencial utilizado pelo preço de mercado } \\
\text { Compatível com o processo contínuo de redução de custos por análise de valor }\end{array}$ \\
\hline
\end{tabular}

FO N T E : adaptado de C lemente e Souza (1998, p.152) 
A metodologia kaizen prioriza a realização de atividades que possam contribuir com melhorias na execução das tarefas. N este caso, facilita a diminuição ou até mesmo a eliminação de custos existentes, estabelecendo também, condições de melhoria e implementação, e análise da limitação dos custos no processo, a fim de atingir respectivas reduções do processo. Essa subtração de custos, muitas vezes, não é constatada pela contabilidade e, por serem relevantes, podem contribuir para a redução ou eliminação de custos específicos.

Incentivar a redução ou eliminação de custos do processo, quando possível, é 0 objetivo do custeio kaizen, uma vez que, este não se preocupa em apurar os custos precisos dos produtos.

Os sistemas baseados na metodologia kaizen possuem várias características importantes. E ntre as quais se destacam:

$\checkmark$ A redução ou eliminação de custos como um compromisso de todos, ou seja, da equipe e não de um único indivíduo;

$\checkmark \quad 0$ cálculo dos custos reais, geralmente, por funcionários da produção, sendo de forma assídua, a equipe junta as informações e as planeja relativa aos custos;

$\checkmark \quad$ Os conhecimentos a respeito dos custos utilizados pelas equipes são conforme 0 ambiente de produção, tendo como meta principal que 0 aprendizado e a melhoria se centralizem nas maiores áreas onde os custos devam ser reduzidos;

$\checkmark$ Os custos-padrão são sempre ajustados, visando refletir as reduções anteriores dos custos reais, buscando melhorias dos custos futuros, isso é, mantêm as inovações confirmadas para processos melhores e explicam um novo estágio para novas melhorias.

\section{Como funciona um sistema baseado na Metodologia Kaizen}

Siqueira (2005) ratifica que Kaizen vem da junção das palavras Kai (que significa mudança) e zen (para melhor), sendo então uma palavra japonesa e a filosofia desse sistema constitui-se de um recurso importante na busca de processos produtivos melhores, mais rápidos e mais enxutos devido à eliminação ou diminuição de tarefas e processos de grande impacto econômico. 
Para o autor acima citado, a aplicação e garantia do sucesso dessa metodologia depende de que sejam assumidos os valores do kaizen pela alta administração da empresa, ou seja, melhorar o processo da empresa de forma contínua deve fazer parte da sua política de qualidade (SI Q U E IRA, 2005).

F arris et al. (2009) destacam que é necessário que a alta gerência absorva essa metodologia; é necessário também que aqueles que são tomadores de decisão, ou seja, a alta administração da empresa dê subsídios, disponibilize recursos para que sejam desenvolvidas atividades para a promoção dos valores adotados, de forma que sejam conhecidos por toda a organização.

São várias as atividades, como: adoção do Programa 5S, ou Programas de Sugestões, C írculos de Q ualidade ou Programas de treinamento e A perfeiçoamento em técnicas baseadas em estatísticas e utilização de ferramentas de qualidade, priorizando também o aperfeiçoamento de técnicas de soluções de problemas, etc. (FARRIS et al., 2009).

Siqueira (2005) ainda cita que todos os funcionários ou colaboradores devem estar cientes e comprometidos com as práticas relativas à melhoria contínua no seu dia a dia, ou seja, deve-se envolver a todos os interessados da organização nas atividades relativas a implantação e a manutenção do Kaizen para que a satisfação do cliente seja plenamente al cançada.

\section{Custo Kaizen e atividades baseadas no ambiente de trabalho}

$\mathrm{N}$ um período determinado pode se verificar dois tipos de atividades referentes à metodologia kaizen: atividades organizadas em relação ao sistema contábil utilizado pela empresa e atividades kaizen (de melhoria nas atividades da produção) no ambiente de trabalho. E stas, por sua vez, buscam eliminar as perdas da fábrica e outros ambientes de trabalho, enquanto as atividades organizadas com base no sistema de contabilidade determinam os objetivos que incentivam as atividades kaizen.

Segundo Shingo (1991), para que haja uma diminuição relevante dos custos de produção efetiva, deve-se analisar e ponderar os desperdícios e perdas, uma vez que eles estão relacionados entre si no decorrer do processo e numa organização complexa. E ssa situação poderá ser ocultada, prejudicando assim a redução ou eliminação dos custos. 
Shingo (1991) complementa que os desperdícios e perdas relativos à produção se dividem nas seguintes categorias:

1) D esperdício de Superprodução: desperdício que ocorre quando se produz esperando que os produtos sejam requisitados futuramente, isto é, produzir grande quantidade, sem saber se haverá demanda para toda a produção;

2) Desperdício de Espera: possuir filas de matérias a serem processados gerando grandes taxas relativas a utilização dos equipamentos de produção.

3) Desperdício de Transporte: transporte e movimentação devem ser otimizados pois não acrescentam valor ao produto, tendo em vista que são necessários em relação ás restrições das instalações e processo que prescrevem distâncias relevantes durante o processamento do material.

4) D esperdício de Processamento: entende-se como um procedimento não aperfeiçoado, ou melhor, há tarefas ou etapas no decorrer do processo que não acrescenta qualidades que venham a valorizar o produto final.

5) Desperdício de M ovimento: a maneira como o operador, as máquinas, ferramentas e os materiais se relacionam durante o processo podem resultam em grandes desperdícios vigentes nas mais diversas execuções do processo produtivo.

6) Desperdício de Produzir Produtos D efeituosos: causados pela falta de qualidade. Produtos com defeitos ou de má qualidade quer dizer, perda de matérias, uso incorreto de equipamentos, desqualificação da mão de obra e também armazenagem e transferência de matérias defeituosas, produtos com necessidade de revisão, etc.

7) Desperdício de Estoques: Relaciona-se estreitamente com os demais desperdícios.

\section{A s perdas são:}

1) Perda Primária: A quantidade excedente de recursos produtivos como funcionários, equipamentos ou estoque, ocasiona desnecessários custos de mão de obra, depreciação de equipamentos e financeiros;

2) Perda Secundária: É considerada a pior perda, pois é relativa à superprodução em quantidade ou superprodução devido à antecipação sem que se tenha certeza de demanda; 
3) Perda Terciária: A grande quantidade de estoque que causa custos financeiros e custos de oportunidade;

4) Perda Q uaternária: Significativa quantidade de transporte em excesso, grande quantidade de estoque, gerando custos administrativos e de manutenção em relação à qualidade.

Siqueira (2005) mostra o kaizen como um complemento às práticas de reengenharia. Por meio da inovação, a reengenharia proporciona a melhoria, traz mudanças dos processos utilizados. 0 kaizen proporciona o melhoramento, eliminando os problemas constatados nos processos vigentes.

\section{Conclusão}

Foi evidenciada a real necessidade em estratificar os custos inerentes ao funcionamento da capacidade produtiva e continuidade do negócio. Independente da metodologia aplicada para ganhos de eficiência e minimização de desperdícios, é indispensável que as empresas disponibilizem capital para investimento e estudos mais aprofundados de forma a garantir a manutenção da competitividade no mercado onde as mesmas encontram-se inseridas.

$\mathrm{N}$ ão obstante, a metodologia Kaizen surge como uma alternativa para que as empresas possam alavancar seus níveis de produtividade. Foi observado que na elaboração de um mapeamento de fluxo de valor, é possível constatar diversas oportunidades de ganho real, seja no rearranjo dos processos internos de produção, seja na diferenciação e diversificação dos produtos oferecidos no mercado.

Portanto, no que se refere à excelência da metodologia Kaizen, ela atua ativamente como um meio importante para redução de custos, se tornando um empreendimento interessante para aperfeiçoar a gestão de custeio no meio empresarial. E m suma, essa metodologia é capaz de proporcionar mudanças na execução do trabalho que serão refletidas no al cance de melhores resultados. 


\section{Referências}

A G N D A L , H enrik, N I LSSO N, U If. Interorganizational costmanagement in theexchange process. M anagement A ccounting R esearch, 2009.

CLE M E NTE, A demir; SO UZA , A Iceu. C usto D esempenho. São Paulo: E ditora F utura, 1998.

CO O PER, R obin, SL A G M UL DER, R egine. Redução de custos com Inteligência. Revista H SM M anagement. São Paulo, 2003.

FARRIS, Jennifer, A KE N, Eileen, D O O LE N , T oni, W ORLEY, June. Critical success factors for human resource outcomes in K aizen events: A $n$ empirical study. Int. J. Production E conomics, 2009.

FE RRE IRA, Ricardo J. Contabilidade de C ustos T eoria equestões comentadas. Rio de Janeiro: E d. Ferreira, 2007.

I M A I, M asaaki. Kaizen: A estratégia para o sucesso competitivo. São Paulo: E ditora: Imam, 1994.

LEITE , G ildo. A dministração da produção: A evolução com a ferramenta de melhoria contínua no Japão. R evista T écnica IPE P, São Paulo, 2007.

M E G LI O RIN I, E vandir. Custos. São Paulo: M akron B ooks, 2001.

M ESQ UIT A, M elissa, A L LIPRA DIN I, D ário. Competências essenciais para a melhoria contínua da produção. R evista G estão \& Produção. São Paulo, 2003.

M O N D E N, Y asuhiro. Sistema de redução de custos, custo- alvo e austo- kaizen. São Paulo: Bookman, 1999.

SA KU RA I, M ichiharu. Gerendamento integrado de custos. São Paulo: A tlas, 1997

SA N T OS, Joel José. M anual de contabilidade e análise de custos. São Paulo: A tlas, 2017. 
SH IN G O , S. Study of T oyota Production System from I ndustrial E ngineering View point. Tokyo, Japan M anagement A ssociation, 1991.

SI Q U E I R A , Juliana. (2005). 0 sistema de custos como instrumento de apoi o ao processo decisório: U m estudo multicaso em indústrias do setor metal-mecânico da R egião N oroeste do E stado do R io G rande do Sul. D issertação de M estrado apresentada à Universidade R egional do N oroeste do E stado do Rio G rande do Sul - UNIJUí, como requisito para obtenção do título de $M$ estre em $D$ esenvolvimento, $G$ estão e C idadania. 